\title{
Extrapolação das relações solo-paisagem a partir de uma área de referência
}

\author{
Extrapolation of soil-landscaperelationship from a reference area
}

\author{
Alexandre ten Caten ${ }^{\mathrm{I}}$ Ricardo Simão Diniz Dalmolin ${ }^{\mathrm{I}}$ Fabrício de Araújo Pedron $^{\mathrm{II}}$ \\ Maria de Lourdes Mendonça Santos ${ }^{\mathrm{II}}$
}

\section{- NOTA -}

\section{RESUMO}

O objetivo deste estudo foi aplicar uma técnica automatizada de mapeamento de solos a partir de modelos preditivos ajustados em uma área de referência $e$, posteriormente, aplicados em áreas próximas de mesmas relações solo-paisagem. Modelos lineares generalizados foram desenvolvidos utilizando-se de nove atributos de terreno, derivados de um Modelo Digital de Elevação, como covariáveis preditoras e classes de solos, obtidas em um levantamento convencional, como variáveis dependentes. Os modelos foram capazes de distinguir as três principais formas da paisagem local. Classes de solos de pedogênese intimamente ligada às covariáveis preditoras obtiveram os melhores resultados. $O$ mapa de solos gerado apresentou uma reprodutibilidade de $46,12 \%$ e uma exatidão de $21,06 \%$.

Palavras-chave: pedometria, mapeamento digital de solos, regressão logística múltipla.

\section{ABSTRACT}

The aim of this study was to apply an automated technique of soil mapping from predictive models developed at a reference area, into nearby areas of the same soil-landscape relationships. Generalized linear models were developed using nine terrain attributes derived from a digital elevation model as covariate predictors, with soil classes, obtained from a conventional soil survey, as dependent variables. The models were able to distinguish the three main forms of the local landscape. Soil classes with pedogenesis intimately tied to the predictive covariates obtained the best results. The soil maps generated, showed a reproducibility of $46.12 \%$ and an accuracy of $21.06 \%$.
Key words: pedometric, digital soil mapping, multinomial logistic regression.

O conhecimento da distribuição geográfica dos solos é fundamental para o adequado uso e preservação deste recurso natural tão lentamente renovável. Contudo, são poucos os municípios que contam com levantamentos de solos em escala semidetalhada ou maiores, fundamentais para estudos e planejamento de bacias hidrográficas, projetos de assentamentos e propriedades rurais (DALMOLIN et al., 2004). A grande demanda por informação acerca da distribuição espacial de propriedades e classes de solos tem impulsionado a aplicação da abordagem quantitativa para a predição espacial e o Mapeamento Digital de Solos (MDS). A técnica e ciência do MDS busca criar e disponibilizar a informação espacial sobre o solo através de métodos de observação em campo e laboratoriais, associados a sistemas de inferência com base nos fatores de formação dos solos (MCBRATNEY et al., 2003).

Uma das estratégias utilizadas no MDS é a extrapolação das relações solo-paisagem de uma área mapeada, definida como área de referência, para regiões onde estas relações ainda não são bem conhecidas. $\mathrm{O}$ método baseado na área de referência assume que é possível delimitar pequenas regiões naturais, as quais

Instituto Federal Farroupilha, Campus Júlio de Castilhos, Júlio de Castilhos, RS, Brasil.

IIDepartamento de Solos, Centro de Ciências Rurais (CCR), Universidade Federal de Santa Maria (UFSM). Av. Roraima, 1000, 97105-900, Santa Maria, RS, Brasil. E-mail: dalmolinr@pq.cnpq.br. Autor para correspondência.

IIICentro Nacional de Pesquisa de Solos, Rio de Janeiro, RJ, Brasil. 
irão conter um finito número de classes de solos formando um padrão reconhecível e repetidamente distribuído. Consequentemente, uma área de referência (representativa da região), poderia ser suficiente para identificar todas as classes de solos em áreas maiores e estabelecer suas relações espaciais (LAGACHERIE et al., 1995).

Assumindo que os mapas de solos contêm as relações entre o solo e o ambiente da área mapeada e que podem ser utilizados como áreas de referência, este estudo teve como objetivo realizar um mapeamento convencional de solos e sua posterior extrapolação para áreas próximas onde as mesmas relações solopaisagem são válidas.

A área de estudo localiza-se no município de São Pedro do Sul, situado na região central do Estado do Rio Grande do Sul (KLAMT et al., 2001). Como a área de estudo apresenta pelo menos duas superfícies geomórficas muito distintas quanto aos processos de formação do solo, uma ligada ao Rebordo do Planalto e outra à Depressão Central, foi realizada a fotointerpretação e checagem a campo nestas duas áreas, as quais constituíram a área de referência para esse estudo (Figura 1A). As classes de solos foram identificadas ao nível de subordem em escala semidetalhada de 1:50.000 a partir do mapa produzido por KLAMT et al. (2001). Em 56 locais sobre a paisagem, verificaram-se as classes de solo presentes ao nível de subordem, bem como, coletaram-se coordenadas para posterior verificação da exatidão dos mapas preditos. A qualidade dos mapas gerados foi avaliada pelo índice Kappa.

Para representar as condições ambientais de formação do solo na área do estudo, foram utilizados atributos de terreno derivados do Modelo Digital de Elevação (MDE) obtido a partir dos dados do Shuttle Radar Topography Mission (SRTM). O MDE foi interpolado para uma resolução espacial de 50 metros, utilizando a função Interpolar para Raster do programa ArcGIS 9.3. Esse mesmo programa foi utilizado para a geração do mapa de classes de solos, conforme detalhado por TEN CATEN et al. (2009).

Os nove atributos de terreno ELEV (Elevação), DECL (Declividade), DIST (Distância à Drenagem), CPLN (Curvatura Planar), CPRF(Curvatura de Perfil), RADI (Radiação relativa disponível), LNAC (Logaritmo natural da área de contribuição), IUT (Índice de umidade topográfica) e CTS (Capacidade de transporte de sedimento), foram gerados de acordo com o procedimento detalhado por WILSON \& GALLANT (2000). O modelo estatístico utilizado para a extrapolação das classes de solos a partir das áreas mapeadas foi o de Regressão Logística Múltipla (RLM).
De acordo com CHATTERJEE \& HADI (2006), a RLM não tem qualquer exigência para sua aplicação a respeito da distribuição das variáveis explicativas, não havendo necessidade de ter distribuição normal, correlação linear, medidas em mesma escala ou homogeneidade de variância. A aplicação de RLM para o mapeamento de solos já foi demonstrada por HENGL et al. (2007) e por FIGUEIREDO et al. (2008).

As classes Solos Hidromórficos (Planossolos e Gleissolos), Argissolos BrunoAcinzentados, Argissolos Vermelhos e Neossolos Litólicos foram espacializadas, de modo semelhante ao que se encontra nos mapas de referência e, consequentemente, ao modelo mental desenvolvido pelo pedólogo durante o mapeamento da área de estudo (Figura 1B). As classes de solos Argissolos VermelhosAmarelos e Cambissolos Háplicos não foram preditas pelos modelos, possivelmente devido à sua pequena representatividade na área do mapeamento convencional, de apenas 5,4 e 2,4\%, respectivamente. Para HENGL et al. (2007) o mapeamento preditivo deve ser realizado a partir de uma representatividade mínima de cada classe de solo, caso contrário, corre-se o risco de os modelos não capturarem toda a complexidade do ambiente de formação do solo da área a ser mapeada.

A avaliação da qualidade dos mapas gerados demonstra que para a classe dos Neossolos Litólicos obteve-se o maior valor de Acurácia do Mapeador (AM), com um valor acima de $98 \%$. Esse elevado valor está relacionado ao fato de que as nove covariáveis preditoras utilizadas para gerar os modelos são derivadas do fator de formação relevo. $\mathrm{O}$ relevo tem forte influência na formação dos Neossolos Litólicos, típicos de áreas de grande declividade. Possivelmente, as demais classes de solos teriam a sua inferência melhorada caso fossem utilizadas variáveis preditoras como geologia e uso da terra, diversificando a representatividade dos demais fatores de formação do solo. Quanto à capacidade do modelo preditivo em reproduzir o mapa da área de referência, obteve-se uma reprodutibilidade de $46,12 \%$, ou seja, obteve-se a mesma classe de solo entre os mapas de referência e o predito (Tabela1). FIGUEIREDO et al. (2008) reportaram valores de índice Kappa de 36,63\% quanto à capacidade de um modelo preditivo por RLM em reproduzir um mapa de solos dos municípios gaúchos de Ibirubá e Quinze de Novembro, utilizado para treinar os modelos.

Entre as classes preditas, verifica-se que o Argissolo Bruno-Acinzentado, com uma proporção relativa de $23,29 \%$ da área utilizada para treinar os modelos, apresentou um valor de classificação correta (AM) de 50,05\%. Para o Argissolo Vermelho, com uma área correspondente a 40,15\% do total, foi verificada 

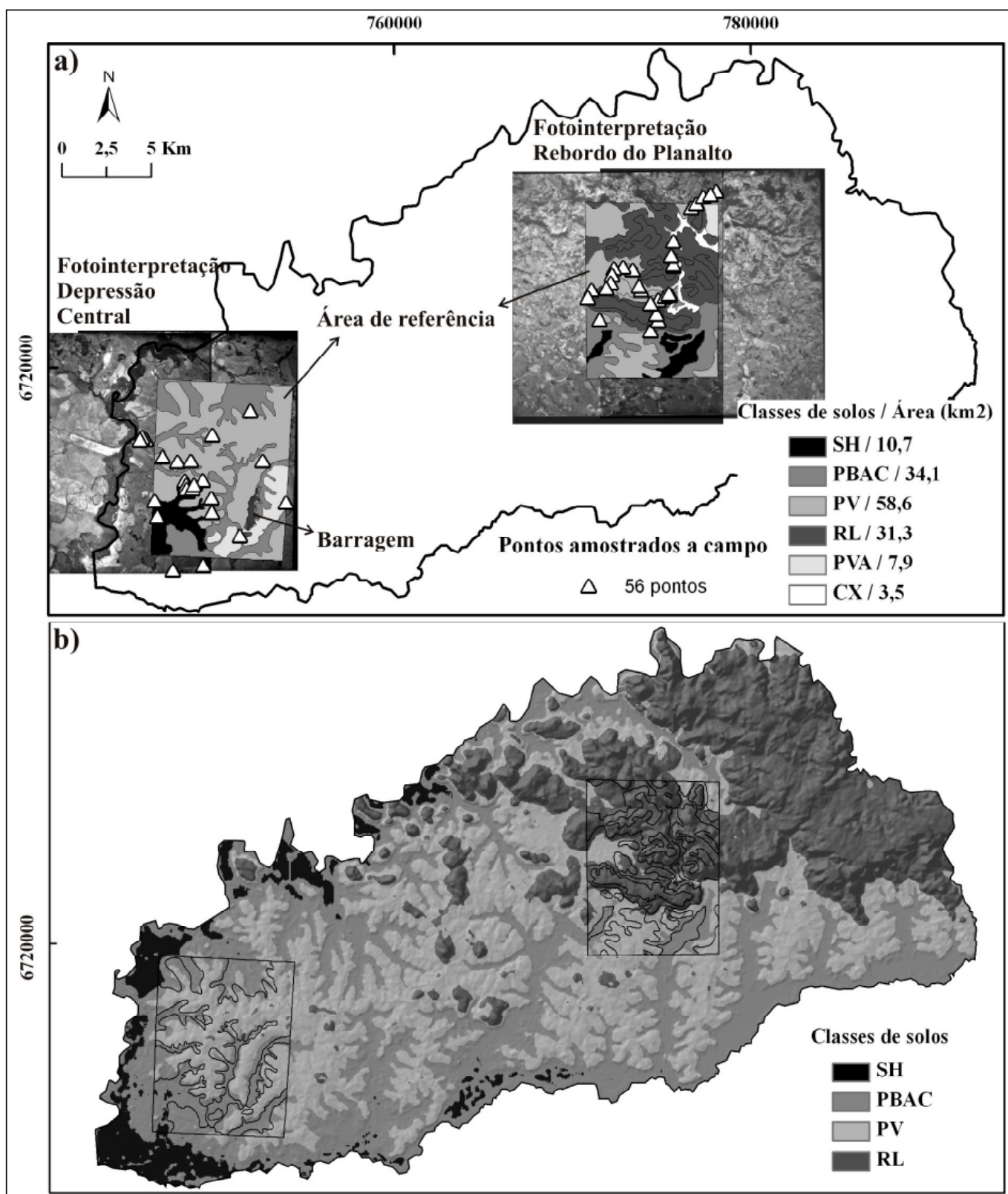

Figura 1 - a) localização da área de referência no município de São Pedro do Sul. b) mapa de solos predito pela extrapolação das relações solo-paisagem, com polígonos de solos da área de treinamento sobrepostos. Coordenadas no sistema UTM Fuso 21, Datum SIRGAS2000. Cambissolo Háplico (CX), Argissolo Vermelho (PV), Argissolo Vermelho-Amarelo (PVA), Neossolo Litólico (RL), Argissolo BrunoAcinzentado (PBAC) e Solo Hidromórfico (SH).

uma acurácia do mapeador de 54,15\%. Esses resultados demonstram haver uma relação entre a proporção relativa de cada classe de solo e a capacidade dos modelos logísticos em espacializá-las.

Na tabela 1, observa-se uma acurácia de $21,06 \%$, sendo este valor até 15 pontos percentuais menor do que os valores reportados por HENGL et al. (2007). Essa baixa acurácia pode ter origem na complexidade inerente à distribuição espacial do solo sobre a paisagem, o que torna a predição da distribuição espacial de classes de solos uma tarefa a ser mais investigada. Contudo, fica demonstrada a capacidade de se gerar a informação sobre a distribuição geográfica das classes de solos para regiões onde esta informação ainda não esteja disponível. 
Tabela 1 - Matriz de erros entre a área de referência, observações a campo e o mapa predito produzido a partir dos atributos do terreno como variáveis dos modelos logísticos. Acurácia do mapeador (AM), acurácia do usuário (AU) e acurácia geral (AG) e índice kappa $(\mathrm{K})$.

\begin{tabular}{|c|c|c|c|c|c|c|c|c|}
\hline \multirow{2}{*}{ Mapa predito } & \multicolumn{8}{|c|}{ - } \\
\hline & SH & PBAC & PV & RL & PVA & $\mathrm{CX}$ & Total & $\mathrm{AU}(\%)$ \\
\hline SH & 0,46 & 0,30 & 0,00 & 0,00 & 0,00 & 0,00 & 0,76 & 60,53 \\
\hline PBAC & 6,02 & 13,61 & 7,29 & 0,00 & 1,42 & 0,00 & 28,34 & 48,02 \\
\hline PV & 1,01 & 8,60 & 26,32 & 0,30 & 4,35 & 0,40 & 40,99 & 64,21 \\
\hline RL & 0,15 & 0,76 & 5,26 & 21,41 & 0,00 & 2,33 & 29,91 & 71,58 \\
\hline PVA & 0,00 & 0,00 & 0,00 & 0,00 & 0,00 & 0,00 & 0,00 & 0,00 \\
\hline $\mathrm{CX}$ & 0,00 & 0,00 & 0,00 & 0,00 & 0,00 & 0,00 & 0,00 & 0,00 \\
\hline Total & 7,64 & 23,28 & 38,87 & 21,71 & 5,77 & 2,73 & 100,00 & \\
\hline $\mathrm{AM}(\%)$ & 6,02 & 58,46 & 67,71 & 98,62 & 0,00 & 0,00 & & \\
\hline $\mathrm{AG}(\%)$ & 61,79 & & $\mathrm{~K}(\%)$ & 46,12 & & & & \\
\hline Mapa predito & $\mathrm{SH}$ & PBAC & PV & $\begin{array}{c}\text { vações } \\
\text { RL }\end{array}$ & $\begin{array}{l}\text { o (exati } \\
\text { PVA }\end{array}$ & $\mathrm{CX}$ & Total & AU(\%) \\
\hline $\mathrm{SH}$ & 5,36 & 0,00 & 0,00 & 0,00 & 0,00 & 0,00 & 5,36 & 100,00 \\
\hline PBAC & 10,71 & 5,36 & 3,57 & 0,00 & 0,00 & 0,00 & 19,64 & 27,29 \\
\hline PV & 1,79 & 3,57 & 23,21 & 1,79 & 10,71 & 0,00 & 41,07 & 56,51 \\
\hline RL & 0,15 & 1,79 & 16,07 & 5,36 & 3,57 & 7,14 & 33,93 & 15,80 \\
\hline PVA & 0,00 & 0,00 & 0,00 & 0,00 & 0,00 & 0,00 & 0,00 & 0,00 \\
\hline $\mathrm{CX}$ & 0,00 & 0,00 & 0,00 & 0,00 & 0,00 & 0,00 & 0,00 & 0,00 \\
\hline Total & 17,86 & 10,71 & 42,86 & 7,14 & 14,29 & 7,14 & 100,00 & \\
\hline $\operatorname{AM}(\%)$ & 30,01 & 50,05 & 54,15 & 75,07 & 0,00 & 0,00 & & \\
\hline $\mathrm{AG}(\%)$ & 39,29 & & $\mathrm{~K}(\%)$ & 21,06 & & & & \\
\hline
\end{tabular}

Cambissolo Háplico (CX), Argissolo Vermelho (PV), Argissolo Vermelho-Amarelo (PVA), Neossolo Litólico (RL), Argissolo BrunoAcinzentado (PBAC) e Solo Hidromórfico (SH).

Conclui-se que o Mapeamento Digital de Solos a partir de uma área de referência pode ser utilizado como alternativa ao mapeamento convencional para dimensões mais extensas da paisagem. A seleção de áreas de referência representativas da paisagem a ser mapeada é uma fase crucial para a adequada extrapolação das relações solopaisagem, além da seleção de variáveis ambientais, as quais tenham forte relação com a pedogênese dos solos a serem mapeados. Futuros estudos devem buscar avaliar a dimensão espacial da validade das extrapolações feitas a partir de uma área de referência.

\section{AGRADECIMENTOS}

Os autores agradecem a Coordenação de Aperfeiçoamento de Pessoal de Nível Superior (CAPES), pela bolsa de Mestrado concedida ao primeiro autor e ao Conselho Nacional de Desenvolvimento Científico e Tecnológico (CNPq), pelo financiamento e pela Bolsa PQ concedida ao segundo autor.

\section{REFERÊNCIAS}

CHATTERJEE, S.; HADI, A.S. Regression analysis by example. 4.ed. New York: John Willey \& Sons, 2006. 375p.
DALMOLIN, R.S.D et al. Relação entre as características e o uso das informações de levantamentos de solos de diferentes escalas. Ciência Rural, v.34, n.5, p.1479-1486, 2004. Disponível em: <http://www.scielo.br/scielo.php?script=sci_arttext\&pid=S0103$84782004000500023 \& \operatorname{lng}=$ en\&nrm=iso >. Acesso em: 20 out. 2010. doi: 10.1590/S0103-84782004000500023.

FIGUEIREDO, S.R. et al. Uso de regressões logísticas múltiplas para mapeamento digital de solos no planalto médio do RS. Revista Brasileira de Ciência do Solo, v.32, n. esp, p.2779-2785, 2008. Disponível em: <http://www.scielo.br/ scielo.php?script =sci_arttext \& pid = S $0100-$ $06832008000700023 \& \operatorname{lng}=\mathrm{en} \& \mathrm{nrm}=\mathrm{iso}>$. Acesso em: 20 out. 2010. doi: 10.1590/S0100-06832008000700023.

HENGL, T. et al. Methods to interpolate soil categorical variables from profile observations: Lessons from Iran. Geoderma, v.140, n.4, p.417-427, 2007. Disponível em: <http://www.sciencedirect.com/science/article/B6V674NPHMTC-3/2/38c5c93ffa96518abae30ea544c18971>. Acesso em: 20 out. 2010. doi: 10.1016/j.geoderma.2007.04.022.

KLAMT, E. et al. Solos do Município de São Pedro do Sul. Santa Maria: Departamento de Solos/CCR/UFSM, 2001. $96 \mathrm{p}$.

LAGACHERIE, P. et al. A soil survey procedure using the knowledge of soil pattern established on a previously mapped reference area. Geoderma, v.65, n.3-4, p.283-301, 1995. Disponível em: <http://www.sciencedirect.com/science/article/ 
B6V67-4002F6M-7/2/5717e53bd7d6951 febd248471351 eff3>. Acesso em: 20 out. 2010. doi: 10.1016/0016-7061(94)00040-H.

MCBRATNEY, A.B. et al. On digital soil mapping. Geoderma, v.117, n.1-2, p.3-52, 2003. Disponível em: <http:// www.sciencedirect.com/science/article/B6V67-496FTGD-1/2/ 81065d61583bf2273a3e4deb685f6c61>. Acesso em: 20 out. 2010. doi: 10.1016/S0016-7061(03)00223-4.
TEN CATEN, A. et al. Mapeamento digital: probabilidade associada à distribuição espacial de classes de solos. Revista Geomática, v.4, n.2, p.87-97, 2009. Disponível em: http:// w3.ufsm.br/rgeomatica/page4/08.pdf. Acesso em: 19 fev. 2011.

WILSON, J.P. ; GALLANT, J.C. Digital terrain analysis. In: (Ed.). Terrain analysis: principles and applications. New York: Wiley \& Sons, 2000. Cap.1, p.1-27. 